\title{
ESTUDO DE MOVIMENTAÇÃO DE CORRENTE MULTIFÁSICA EM TUBOS UTILIZANDO FERRAMENTA CFD
}

\author{
F.C.S. SIQUEIRA ${ }^{1}$, I.S. FARIAS ${ }^{2}$, D. MORAES Jr. ${ }^{2}$ e A.S. VIANNA Jr. ${ }^{1}$ \\ ${ }^{1}$ Universidade de São Paulo, Departamento de Engenharia Química \\ ${ }^{2}$ Universidades Santa Cecília, Departamento de Engenharia Química \\ E-mail para contato: ardson@usp.br
}

\begin{abstract}
RESUMO - A descoberta de petróleo na camada do Pré-Sal fez com que a Petrobras invista cerca de 240 bilhões de dólares. Por isso, o estudo de transporte águaóleo é de grande importância. O objetivo aqui é estudar uma corrente água-óleo em um tubo reto e em uma curva, com o intuito de gerar perfis de velocidades, pressões e densidades. Para as simulações desenvolvidas são considerados fluxo 2D, escoamento turbulento, isotérmico, incompressível e no estado estacionário. Foi realizado o estudo da convergência de malha. Os resíduos globais para pressão e velocidade caem pelo menos 2 ordens de grandeza. $\mathrm{O}$ modelo utilizado para representar a interação entre as fases foi o IPSA, que resolve as equações de Navier-Stokes para cada fase. A turbulência foi modelada utilizando o k- $\varepsilon$ padrão e o k- $\omega$. As densidades geradas nas simulações em CFD foram comparadas visualmente com os resultados experimentais obtidos em trabalho anterior para fluxo core-flow. As simulações podem ser consideradas como pré-validadas.
\end{abstract}

\section{INTRODUÇÃO}

Este trabalho visa o estudo de uma corrente multifásica onde há o escoamento de águaóleo, procurando reproduzir o padrão conhecido como Core Annular Flow, onde um anel de água envolve o óleo pesado. Existem registros de patentes com o intuito de reproduzir este fluxo desde 1904, porém a primeira linha de importância foi somente construída em 1970 pela SHELL, com aproximadamente 38 quilômetros de extensão e ficou em operação por 12 anos (Joseph et al., 1997).

Alguns trabalhos prévios já foram feitos, tratando da modelagem de condições de operação como Perda de Carga e Fração Volumétrica. Prada (1999) constatou que o fluxo é estável para certas faixas de velocidades e que apresenta perdas de carga relativamente baixas, sendo assim uma possibilidade de redução de custos. Vara (2001) percebeu que uma das maiores dificuldades é manter a estabilidade do fluxo ao longo de grandes distâncias, com isso formulou um diâmetro mínimo estável para o core annular flow. Joseph et al. (1997) também observou que a estabilidade do fluxo é função da velocidade das fases.

A descoberta de petróleo na camada do Pré-Sal nos estados de São Paulo, Rio de Janeiro e Espírito Santofez com que a Petrobras invista cerca de 240 bilhões de dólares, até 2017, para que haja a produção de 1 milhão de barris/dia (Petrobras, 2014). Portanto, o estudo da movimentaçãode petróleo (óleo) é de grande importância para a situação atual do país. 


\section{FUNDAMENTOS TEÓRICOS}

\subsection{ESCOAMENTO MULTIFÁSICO}

Escoamento multifásico é quando mais de uma fase está escoando simultaneamente (Rosa (2012). Por fase subentende-se uma região do espaço delimitada por uma interface de espessura infinitesimal, que encerra em seu interior material com composição química homogênea, propriedades de transporte e de estado definíveis e que é separável por processos mecânicos.

A forma como a mistura escoa depende da velocidade dos fluídos. Joseph et al. (1997) estudaram diversos escoamentos bifásicos em tubulações horizontais e verticais, sendo possível observar diversos padrões: dispersão de óleo em água; gotas alargadas ou esféricas; bolhas alongadas; pistonado; estratificado; anular; core-flow ou bamboo waves; wavy; core annular flow com dispersão de bolhas e dispersão de água em óleo.

Óleos pesados são altamente viscosos e mais leves que a água, possuindo tipicamente viscosidades de 1000 Poise e uma densidade de $0,99 \mathrm{~g} / \mathrm{cm}^{3}$ na temperatura de $25^{\circ} \mathrm{C}$. Sendo assim, por diferença de densidade, a água tende a ficar nas paredes da tubulação lubrificando o fluxo. Óleos leves com viscosidades abaixo de 5 Poise só conseguem gerar fluxos lubrificados estáveis em emulsões água-óleo.

Joseph et al. (1997) citam que a viscosidade dessas emulsões pode ser bem menores que a do óleo e podem ser independentes para grandes valores. Isso tem motivado a consideração de bombear óleo bruto pesado através de tubulações como emulsões água-óleo e apresenta uma tubulação comercial na indonésia que produz 40 mil barris por dia de $70 \%$ emulsão óleo-água com $50 \mathrm{~cm}$ de diâmetro e $238 \mathrm{~km}$ de comprimento.

\subsection{CFD}

CFD Computational Fluid Dynamics é o nome dado para a ferramenta numérica e computacional que resolve as equações de conservação de grandezas físicas e assim predizer o comportamento de fluxos de gases e líquidos. A prática apresenta baixo custo, já que não requer a construção de experimentos ou plantas piloto (POLIS 2014). Com o auxílio dessa ferramenta,

O software utilizando foi o PHOENICS que é um código de CFD utilizado para o estudo de fluxos, empregando coordenadas Cilíndricas-Polares ou Cartesianas. Ele foi lançado comercialmente em 1981 pela Concentration, Heat and Momentum Limited in Wimbledon. O nome PHOENICS é um acrônimo para Parabolic, Hyperbolic or Eliptic Numerical Integration Codes Series (POLIS, 2014).

\subsection{MODELOS}

O PHOENICS possui diversos modelos para representar inúmeras condições operacionais. Para o presente estudo de caso foram avaliados modelos para turbulência e para sistema multifásico. 


\subsubsection{Multifásico}

Os métodos de simulação presentes no software são o IPSA (Inter-Phase-Slip Algorithm), ASLP (Algebraic SLip Model), SEM (Scalar Equation Method), HOL (Height Of Liquid), GENTRA (GENeral TRAcking).

O IPSA é o método utilizado, onde se resolve as equações de Navier-Stokes para cada fase. Normalmente as duas fases possuem pressões iguais, porém, podem ser diferenciadas caso exista pressão de contato entre partículas sólidas. Também é computada uma fração de volume para cada fase e ainda cada fase pode possuir composição, tamanho de partícula e quantidade de turbulência (POLIS, 2014).

\subsubsection{Turbulência}

Turbulência é o estado do movimento do fluido caracterizado por aparentes vórtices caóticos tridimensionais (Souza et al. 2011). Quando a turbulência está presente no escoamento ela aumenta a dissipação energética, a mistura, a transferência de calor, entre outros. O estudo da turbulência é necessário para melhor compreensão do escoamento, por isso diversos modelos para turbulência foram desenvolvidos, porém os mais usados são os modelos do tipo RANS de

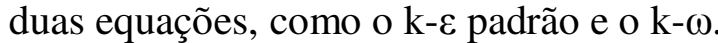

$\mathrm{O}$ k- $\varepsilon$ padrão possui a vantagem de ser de fácil implementação, tem boa convergência e apresenta bom funcionamento para escoamentos simples, como o escoamento em dutos ou escoamento em camada limite. O modelo não funciona muito bem para escoamentos complexos, como, por exemplo, escoamento em degrau (Silveira Neto, 2002).

O k- $\omega$ possibilita maior facilidade de integração na parede e, assim, outra gama de problemas são possíveis de serem solucionados. Mas o modelo apresenta alta sensibilidade a variações das propriedades nas zonas longe da parede e a tendência é uma maior necessidade computacional (Silveira Neto, 2002).

\section{MÉTODO}

O objetivo deste trabalho é estudar uma corrente água-óleo em um tubo reto e uma curva, com o intuito de quantificar parâmetros e estudar perfis de escoamento. As simulações em CFD foram primeiro realizadas para um tudo reto e depois para uma curva. Em todas as simulações foram atendidas a convergência de malha e a redução dos resíduos. Finalmente as simulações foram comparadas com fotos obtidas na unidade experimental previamente estudada (Farias, 2013) e uma validação preliminar foi observada.

Para simulação foi criado uma caixa sólida com propriedades simulares a do aço inoxidável, onde seu volume ocupa todo o sistema; tubos retos concêntricos de diâmetro $0,18 \mathrm{~m}$ e $0,12 \mathrm{~m}$ preenchidos de água e óleo respectivamente; duas curvas concêntricas com diâmetro de $0,18 \mathrm{~m}$ e $0,12 \mathrm{~m}$ preenchidos de água e óleo respectivamente. A Figura 3 demonstra a geometria. 
A simulação baseia-se em um fluxo multifásico de água com densidade de $0,988 \mathrm{Kg} / \mathrm{L}$ e óleo com densidade de $917 \mathrm{Kg} / \mathrm{L}$, em um tubo com uma curva em uma das pontas. Foi aceito convergência quando se tem uma somatória de resíduos em ordem negativa e valores constates das variáveis ao longo da simulação (Rosa, 2001).

A malha que melhor atendeu o sistema é composta por 408.482 células hexaédricas. A técnica PARSOL (Partial Solids) foi usada para melhorar a precisão da simulação de fluxo para as situações em que há um cruzamento oblíquo sólido/líquido nascélulas da grade cartesiana, assim as células são divididas em duas, onde em uma estará o sólido e na outra o líquido.

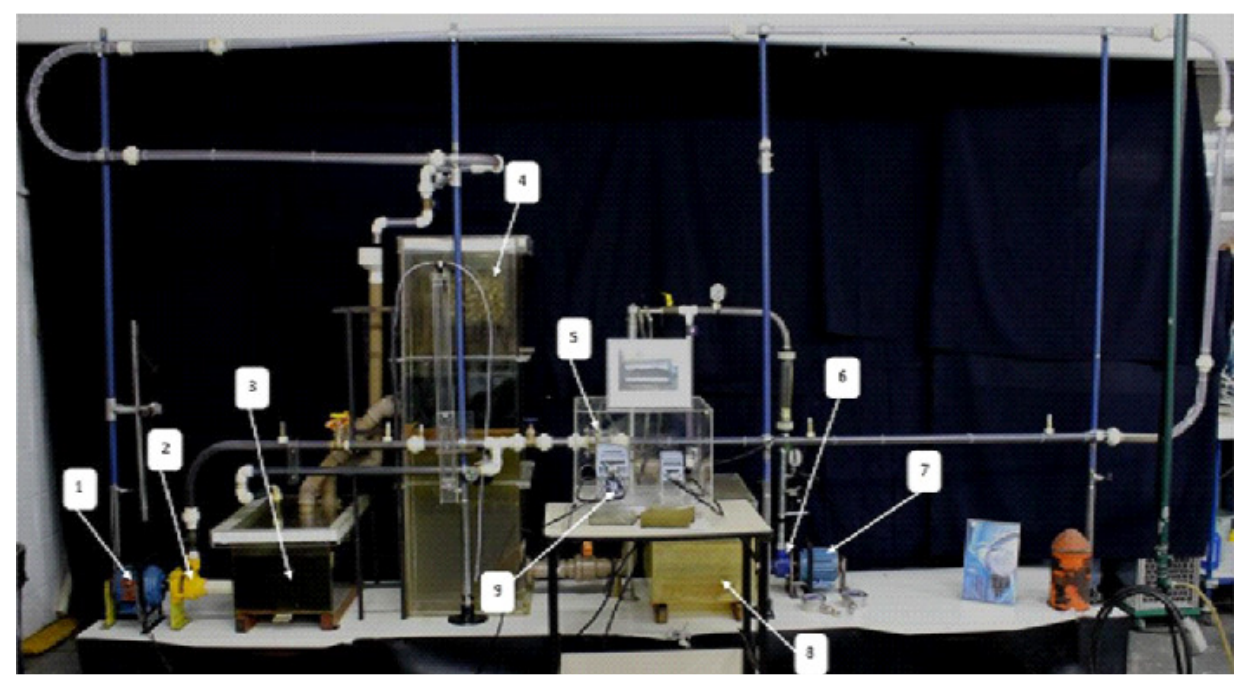

Figura 1 - Planta em escala de laboratório. Legenda: 1 - Motor, 2 - Bomba Regenerativa, 3 - Tanque de óleo, 4 - Tanque água/óleo, 5 - Injetor, 6 - Bomba, 7 Motor de 1HP, 8 - Tanque de água, 9 - Inversor de frequência para os motores.

A unidade experimental apresentada na Figura 1é composta por um motor WEG 22 Plus de 2 HP com rotação nominal de 3385 RPM, ligado à bomba regenerativa Ferrari modelo IDB de 1 CV, com rotação de 3500 RPM e altura manométrica de 50 metros de coluna de água (mca) (Farias, 2013).

\section{DISCUSSÕES E RESULTADOS}

\subsection{Simulações no tubo reto}

Nas simulações foi adotado fluxo 2D, escoamento turbulento, isotérmico, incompressível e no estado estacionário, velocidade de entrada do óleo de $1 \mathrm{~m} / \mathrm{s}$ e da água de $2 \mathrm{~m} / \mathrm{s}$. Para a interface foi aceito que não há transferência de calor e massa, somente transporte de quantidade de movimento. A convergência foi atingida utilizando a ferramenta CONWIZ do Phoenics (2014). Para as simulações utilizando o modelo de turbulência k-ômega, o software apresentou convergência após 15 min e para o k- $\varepsilon 5 \mathrm{~min}$. Os resultados para o primeiro modelo de 
turbulência podem ser vistos nas Figuras 2A e B para velocidade e pressão respectivamente; já para o segundo modelo, as Figuras $2 \mathrm{C}$ e D apresentam os resultados.

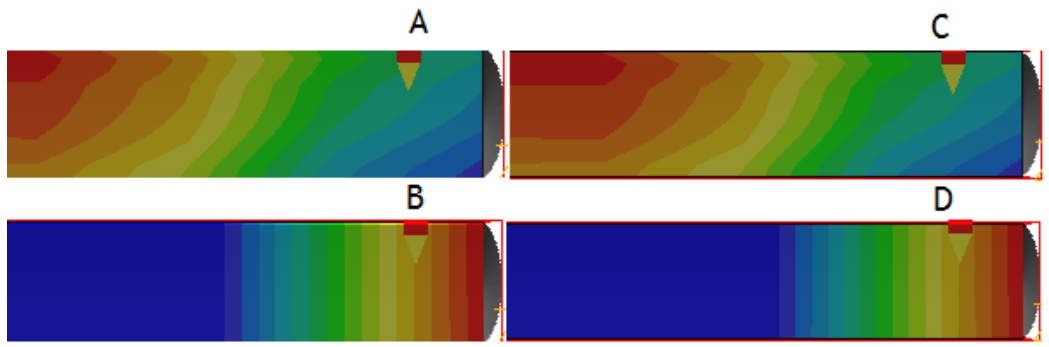

Figura 2 - A) Velocidade para k- $\omega$; B) Pressão para k- $\omega$; C) Velocidade para k- $\varepsilon$; D) Pressão para k- $\varepsilon$.

Os resíduos para as simulações no tubo reto podem ser visto na Tabela 1.

Tabela 1 - Resíduos da simulação usando os modelos k- $\omega$ e k- $\varepsilon$

\begin{tabular}{|l|l|l|l|l|l|l|l|}
\hline & P1 & U1 & V1 & W1 & R2 & K & Ômega \\
\hline k- $\omega$ & $4,410^{-4}$ & $2,310^{-5}$ & $9,610^{-6}$ & $4,810^{-3}$ & $4,410^{-4}$ & $8,710^{-6}$ & $5,110^{-3}$ \\
\hline k- $\varepsilon$ & $8,610^{-4}$ & $2,410^{-5}$ & $8,910^{-6}$ & $5,110^{-3}$ & $8,610^{-4}$ & $8,710^{-6}$ & $1,410^{-5}$ \\
\hline
\end{tabular}

\subsection{Simulações no trecho curvo}

Para as simulações aqui apresentadas foram considerados fluxo $2 \mathrm{D}$, escoamento turbulento, isotérmico, incompressível e no estado estacionário, velocidade de entrada do óleo de $1 \mathrm{~m} / \mathrm{s}$ e da água de $2 \mathrm{~m} / \mathrm{s}$. Para a interface foi considerado que não há transferência de calor e massa, somente transporte de quantidade de movimento. A convergência foi atingida utilizando a ferramenta CONWIZ do Phoenics (2014).

Para as simulações utilizando o modelo de turbulência k-ômega, o software alcançou convergência após 1 hora. Os perfis de velocidade e pressão podem ser vistos nas Figuras 4A e B.

A curvatura da tubulação resulta em modificações tanto no perfil de velocidade quanto na pressão. No raio interno da curva, as pressões são menores do que no raio externo. Observa-se uma região de maior velocidade junto ao raio interno (regiões vermelhas nas Figuras 4A e C).

As velocidades foram reduzidas junto à parede, provavelmente devido à condição de contorno de não deslizamento.

Os resíduos das variáveis para essa simulação estão presentes na Tabela 2. Estes foram menores que o da simulação anterior, para o tubo reto.

Tabela 2 - Resíduos da simulação com os modelos k- $\omega$ e k- $\varepsilon$

\begin{tabular}{|l|l|l|l|l|l|l|l|}
\hline & P1 & U1 & V1 & W1 & R2 & K & Omega \\
\hline $\mathrm{k}-\omega$ & $2,710^{-3}$ & $1,410^{-3}$ & $1,910^{-3}$ & $2,410^{-3}$ & $2,710^{-3}$ & $9,910^{-3}$ & $3,010^{-1}$ \\
\hline $\mathrm{k}-\varepsilon$ & $6,810^{-4}$ & $1,110^{-3}$ & $1,010^{-4}$ & $2,310^{-3}$ & $6,910^{-4}$ & $3,210^{-5}$ & $5,510^{-4}$ \\
\hline
\end{tabular}




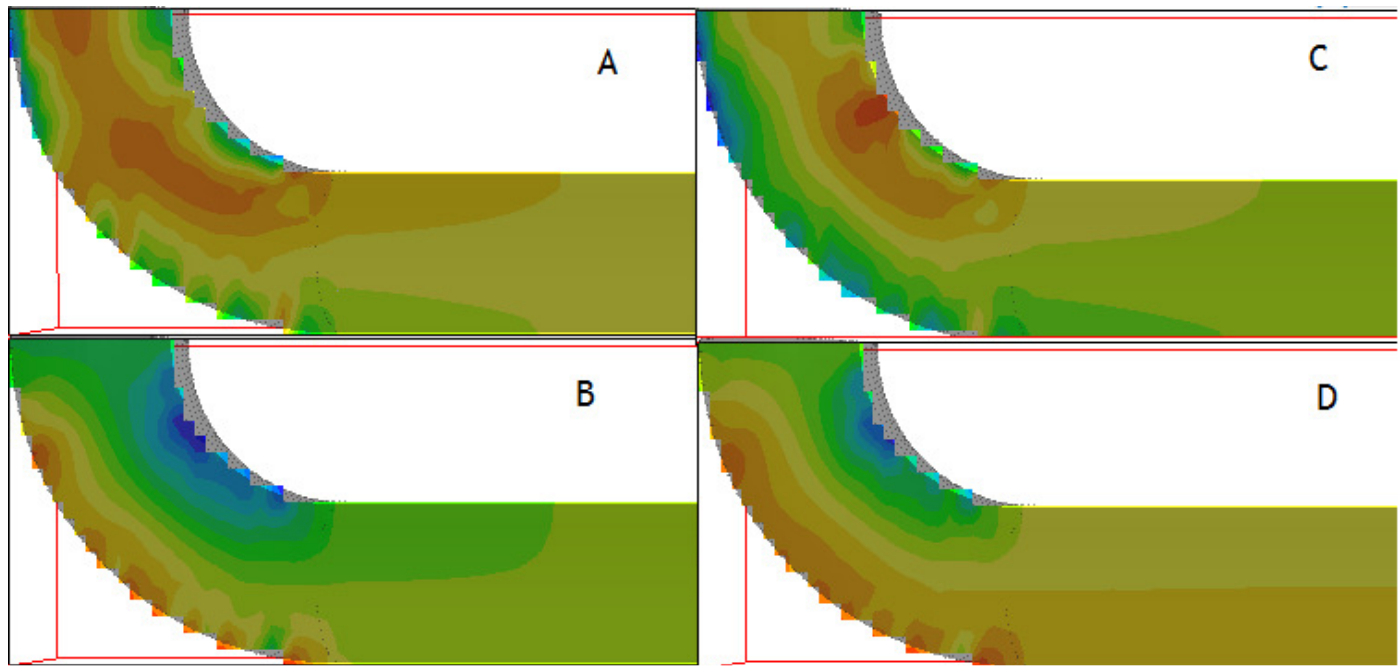

Figura 4 - A) Perfil de Velocidade k- $\varepsilon$; B) Perfil de Pressão k- $\varepsilon$; C) Perfil de Velocidade k$\omega ;$ D) Perfil de Pressão k- $\omega$.

Para as simulações utilizando o modelo de turbulência k- $\varepsilon$, o software alcançou convergência após 10 min. Os perfis de velocidade e pressão podem ser vistos nas Figuras $4 \mathrm{C}$ e D. O perfil de velocidades no centro do tubo e ao longo da posição axial, ponto $0 \mathrm{~m}$ até $2,1 \mathrm{~m}$, que corresponde ao meio da curva estão na Figura 5. Observa-se que a velocidade máxima atingida pelo sistema é de aproximadamente $4 \mathrm{~m} / \mathrm{s}$, sem diferenças significativas para os dois perfis.

$\mathrm{O}$ perfil de densidades para as simulações com $\mathrm{k}-\varepsilon$ e $\mathrm{k}-\omega$ foram muito similares. As Figuras 6 e 7 apresentam os resultados das simulações, onde o óleo (azul) está no centro da tubulação e a água (vermelho) ao seu redor.
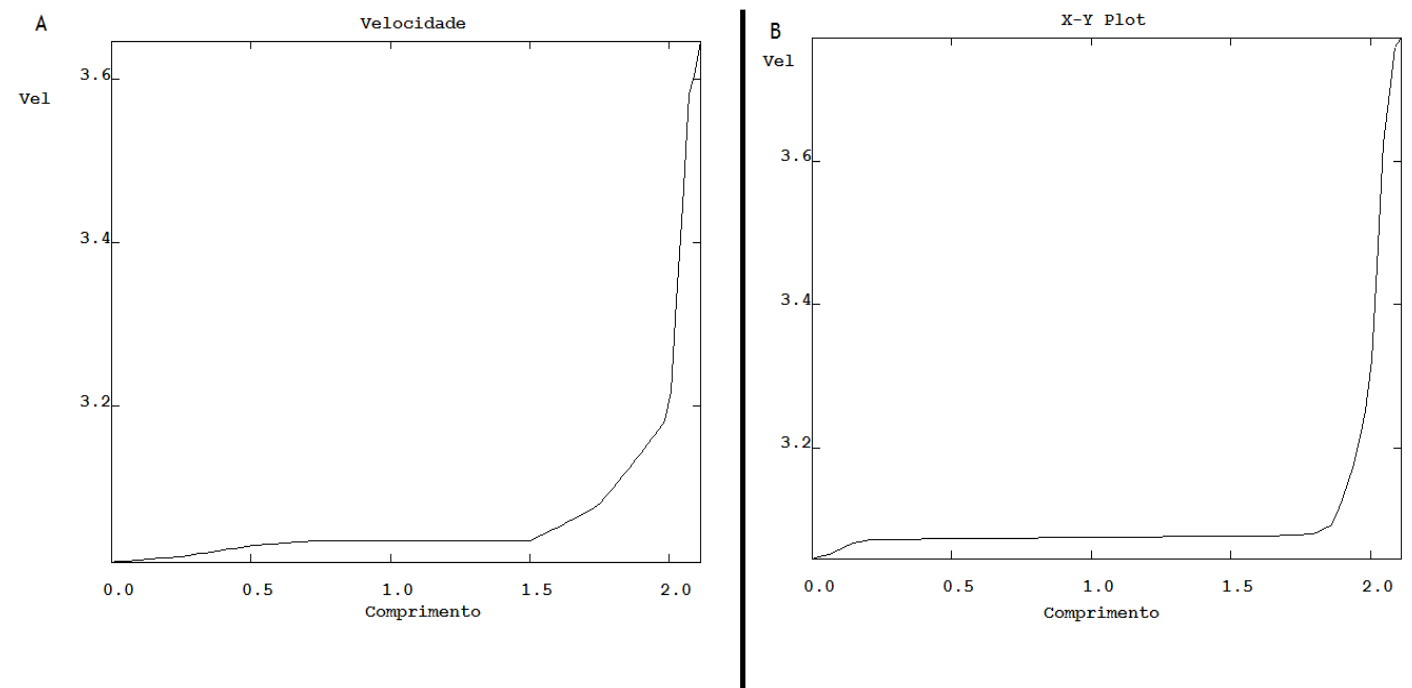

Figura 5 - A) Velocidades usando modelo k- $\varepsilon$; B) Velocidades usando modelo k- $\omega$. 

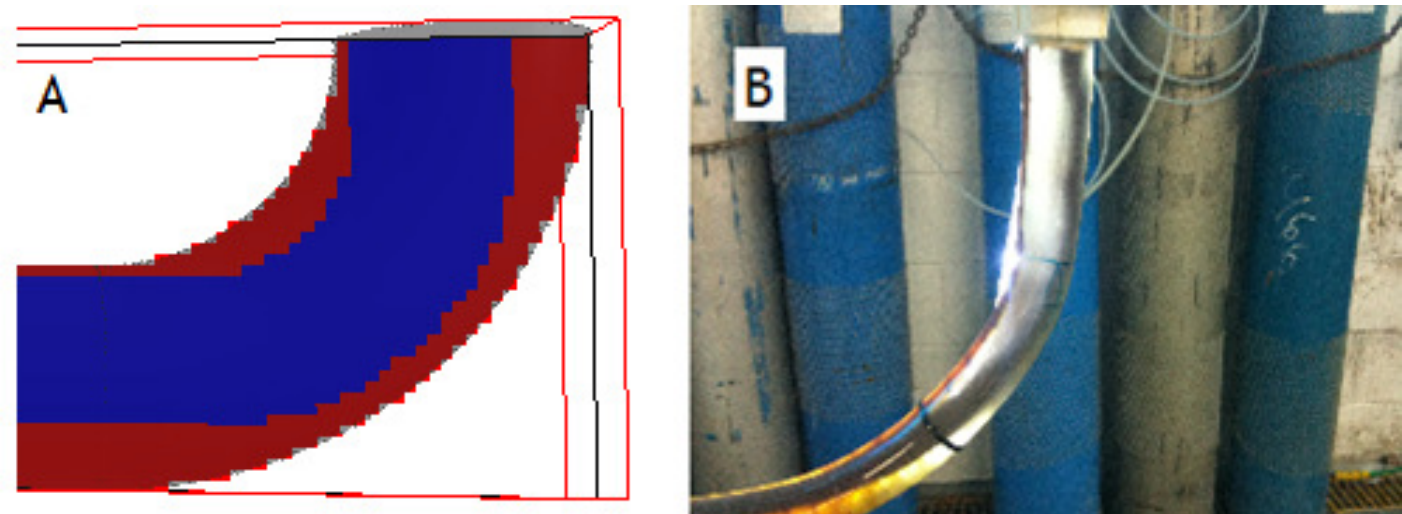

Figura 6 - A) Simulação das densidades na curva; B) Foto do Trecho Curvo do Equipamento.

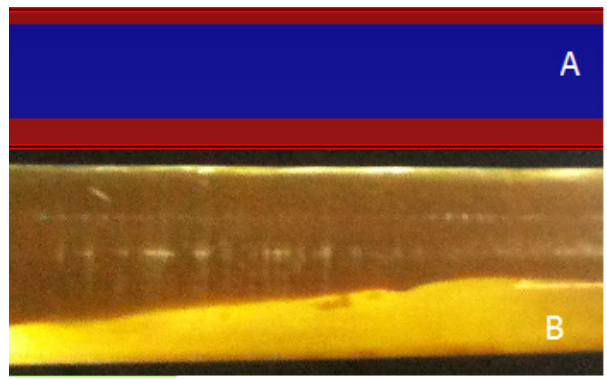

Figura 7 - A) Simulação das densidades no trecho reto; B) Foto do Escoamento no trecho reto.

\subsection{Validação preliminar}

Prada (1999) avaliou uma unidade experimental em escala de laboratório com tubulação vertical de 2,76 cm de diâmetro e utilizou um óleo de 17,6 Pa.s. O core annular flow permitiu reduzir a perda de carga por atrito em mais de 100 vezes, sendo a perda de carga reduzida 93 vezes em comparação ao escoamento monofásico de óleo (Farias, 2013). Nas simulações foi possível observar que a perda de carga para o sistema Água - Óleo foi de $2688 \mathrm{~Pa}$ com uma velocidade máxima de $4,17 \mathrm{~m} / \mathrm{s}$, enquanto que para o sistema bombeando somente óleo, a perda de carga foi de $23.811 \mathrm{~Pa}$ e a velocidade máxima atingida foi de $1,13 \mathrm{~m} / \mathrm{s}$.

Para a efetiva validação dos resultados, seria necessária a utilização de equipamentos sofisticados. Neste trabalho, fez-se uma validação preliminar comparando-se as simulações obtidas aqui e fotos da unidade experimental (Figuras 6 e 7). Pode-se afirmar que houve uma concordância satisfatória tanto para o trecho reto quanto para o trecho curvo. 


\section{CONCLUSÕES}

As simulações obtidas neste trabalho geraram perfis de velocidade, pressão e densidades consistentes com o fenômeno físico. A figura do core annular flow, com água nas bordas e óleo no centro, foi reproduzida.

Em todas as simulações foram observadas a convergência de malha e a redução dos resíduos para todas as variáveis, o que indica que foram atendidas as boas práticas de simulação.

Foi possível observar que no tubo reto a velocidade manteve-se constante ao longo de todo percurso, enquanto que para a curva houve aumento da velocidade no acessório. Houve redução significativa da perda de carga no core annular flow em comparação com o fluxo monofásico de óleo.

As simulações foram previamente validadas com valores da queda de pressão dos estudos prévios de Prada (1999) e com a observação experimental feita por Farias (2013).

\section{REFERÊNCIAS}

FARIAS, I. S., Determinação teórica e experimental do ponto de operação de uma unidade de bombeamento com escoamento monofásico e bifásico (água-óleo), IPT, São Paulo, 2013.

JOSEPH, R., CHEN, K., RENARDY, Y., Core-Annular flows. Annu. Rev. Fluid Mechanics, Vol. 29, pp 65-90, 1997;

PETROBRAS. Plano de Negócios. Disponível em: <http://www.petrobras.com.br>. Acesso em: 15 abril 2014;

PHOENICS On-Line Information System (POLIS), Models. London, England. Disponível em: < http://www.cham.co.uk/>. Acesso em 15 Abril 2014;

PRADA, J. W. V., Estudo experimental do "core annular flow" na Elevação de Óleos Ultraviscosos, Faculdade de Engenharia Mecânica, Universidade Estadual de Campinas, 1999;

ROSA E. S. Escoamento Multifásico Isotérmico. Porto Alegre: Bookman, 2012;

SILVEIRA NETO A., Fundamentos da Turbulência nos Fluidos. Uberlândia: Universidade Federal de Uberlândia, 2002.

SOUZA, J. F. A. Uma revisão sobre turbulência e sua modelagem. Revista Brasileira de Geofísica. v. 29, p. 21-41, 25 mar. 2010.

VARA, R. M. O. Hidrodinâmica do Escoamento Bifásico Óleo Pesado - Água em um Tubo Horizontal. Curso de Engenharia Mecânica, Universidade Estadual de Campinas, 2001. 